\title{
Normkonform, non-konform, ganz normal abweichend
}

\section{Ewa Nowak (Poznań)}

Gesine Drews-Sylla, cheap Elisabeth Dütschke, pilule Halyna Leontiy, sales Elena Polledri (Hrsg.), Konstruierte Normalitäten? normale Abweichungen. VS Research Verlag für Sozialwissenschaften / Springer, Wiesbaden 2010, S. 328.

\section{Das Leben übertrumpft jedes Gesetz}

Mit diesem Spruch wollte der brillante deutsche Rechtsdenker aus der Weimarer Zeit auf die Relativität jeglicher Normierungen aufmerksam machen. Es waren Zeiten, zu denen Europäer sowohl im Westen als auch im Osten ihre üblichen, klaren Orientierungspunkte verloren und sich aus lautem Verirren eifriger denn je einem bald proletarisch, bald faschistisch geprägtem Neubestimmen von ?Rechtem? und ?Unrechtem? gewidmet haben. ?? unsere Zeit duldet keine Zweideutigkeiten? (nach: Kaminskij, ?Störungssignale im sozrealistischen Normensystem?, Konstruierte Normalitäten ? normale Abweichungen, a.a.O., S. 67). In wie fern aber duldet unsere Zeit die Zweideutigkeiten?

In dem frisch erschienenem Band ?Konstruierte Normalitäten ? normale Abweichungen? folgen zweiundzwanzig Autoren und Autorinnen der Radbruch's schen Feststellung. Als Kultur-, Literatur-, Sprach-, Sozial- und Politikwissenschaftler/innen enthüllen sie eine jener Wahrheiten, die den Einen (nennen wir sie Normalitätsvertreter) - immer schon als entsetzlich erscheinen, den Anderen aber? als eben den Anderen (die wir Normalitätsübertreter nennen könnten) ihre conditio transgressiva beleuchten und schon dadurch etwas erleichtern. ?Gemeinsam ist allen Beiträgen, dass sie den Menschen als beständigen Konstrukteur von Normen beschreiben, um mit der (wahrgenommenen) Komplexität und Unsicherheit der heutigen Welt zurecht zu kommen. Dieser Wunsch, eine definierte Normalität zu erschaffen, an der der Einzelne bewusst teilhaben kann, wird jedoch konterkariert durch die Wünsche nach Individualität und Diversität, welche die Normengrenzungen beständig aufweichen und erneut Unsicherheit hervorbringen? (Einleitung, S. 22).

\section{Zu Inhalt, Konstruktion und kulturhistorischer Reichweite des Bandes}

Das Buch ?Konstruierte Normalitäten?? bildet ein reichhaltiges, interdisziplinäres, fast monumentales Sammelwerk, dessen Autorinnen und Autoren zwar dasselbe überzeitliche Phänomen ans Tageslicht bringen, dennoch jede und jeder von ihnen tut es auf eine individuelle Weise. ?Gemeinsam? ist ihnen dennoch das Interesse, welches stets auf den tragischen, destruktiven (auf der anderen Site aber befreienden und konstruktiven) Kampf zwischen institutionalisierter bzw. im 'populären' Bewusstsein stereotypisierter Normalität einerseits und Abweichung andererseits fokussiert ist. Sie revidieren einige kulturelle Topoi und ideologieträchtige Medien (v.a. die Sprache), die einem geschlossenen System (sei es Sozrealismus, sei es Heterosexualität, sei es 'westliche' Marktwirtschaft) jeweils einen Rahmen verleihen, sich aber gleichzeitig gegen die reale Lebensdynamik und Lebenskomplexität wenden. Diese exklusiven, repressiven und schließlich unbrauchbaren Normalitäten ? wie auch deren mentale und institutionelle Genesis - werden im vorliegenden Band meisterhaft bloßgestellt.

Die behandelten Topoi bzw. Narrativa haben die Herausgeberinnen in vier Buchsektionen geteilt. Jede Sektion basiert auf einer eigenen Fragestellung. So werden zuerst die Konstruktion und Dekonstruktion von Normen (bzw. Narration) aus der Perspektive des gesellschaftspolitischen Diskurses betrachtet. In der zweiten (und zum Teil auch in der dritten) Sektion bekommt der Leser einen Überblick, welche Strategien von Aneignung und Normalisierung des Fremden die Literatur erarbeitet hat. Bereits die klassischen Meisterwerke wurden zur Arena der Dialektik zwischen diversen (oft auf tragischer Weise kollidierenden) Denkperspektiven, Bedürfnissen, Lebensstilen etc. die durch Machtinstanzen und die Gesellschaft selbst reglementiert, kontrolliert, delegalisiert, still gelegt oder gar aufgehoben werden. In der Zuflucht literarischer Diskurse und Kunstdarstellungen kommen sie aber wieder zu Wort, werden wieder belebt und erlebt, miteinander kombiniert und auf diese Weise wirkmächtig gemacht. Darin liegt auch die enorme Kraft der Literatur: dass sie das Verstehen des Anderen und das postkonventionelle Denken fördert, indem sie den Leser zur eigenen dekonstruktivistischen Aktivität animiert. So können wir bei Petrarca (Polledri: ?Petrarca spricht Deutsch??) und bei Kapuscinski (Vojta: ?Der Fremde als Abweichung vom Normalen?) denselben überzeitlichen Kampf zwischen dem Eigenen und dem Fremden, und ihre Versöhnung erfahren. Die beiden Elemente erscheinen nur in einer kulturell variierenden Inszenierung. Kontinuierlich thematisieren die in dritter Buchsektion gesammelten Beiträge das Eigene und das Fremde im Kontext der Transmigration, die das Leben zwischen Auswanderung und Einwanderung, zwischen Exklusion und Inklusion bedeutet. Schließlich kommen die Autorinnen und Autoren wieder auf eine (diesmal multinationale, multisprachige, multikulturelle) Gesellschaft, die umso mehr nach der neuen Norm und Normalität suchen muss.

\section{Zur Expressivität des Sammelwerkes}

In allen vier Sektionen wird jeweils das Individuelle dem Gesellschaftlichen, das Heterogene dem Homogenen, das Abweichende dem Normalen usw. entgegengesetzt. Die darauf folgende Dialektik wird nach dem Plan hintergefragt: wo ist das Problem zu lokalisieren? Was ist hier eine Position? eine Negation? Welche Selbsterhaltungs- oder Versöhnungsstrategie wurden bereits 
entwickelt?Die Dialektik wird so gründlich hintergefragt, bis die beiden Gegensätze (das Eigene und das Fremde) in voller Schärfe samt psychologischer Vertiefung des jeweiligen Protagonisten klargestellt bzw. bloßgestellt werden. Ein Paradebeispiel für diese analytische Kunst ist der von Kaminskij sezierte 'Fall Platonov' (?Störungssignale im sozrealistischen Normensystem?.) Den Autorinnen und Autoren liegt es sichtlich am Herzen, den Leser für die hochaktuellen Konflikte zu sensibilisieren, die für ein Individuum entweder eine gespaltene Identität (ein Doppelleben, ein anarchisches Leben) oder ein instabiles Leben am Rande oder bestenfalls ?im Dazwischen? bedeuten (Baumberger, ?Literaturen der Transmigration?, S. 154). So wird der Leser durch spektakuläre Dokumente, Interviews, literarische Bilder, Filmszenarien und Popkultur-Symbole emotional aktiviert und zur Teilnahme an der Dekonstruktion kultureller/gekünstelter Narrativa sowie Stereotypen eingeladen. Dies ermöglicht dem Leser ?den Anderen? über die Einschränkungen und Ausgrenzungen hinweg zu reflektieren. Die Lektüre konfrontiert den Leser sowohl mit der Erfahrung des Konflikts zwischen dem Eigenen und dem Anderen als auch mit der Sprach- und Reflexionskompetenz (sowie Selbstreflexions- und Metareflexionskompetenz), die zu gegenseitigem Verstehen, Kommunikation, Integration und Inklusion beitragen können. Der Leser kann sich wie ein Reisender fühlen, der dem Ortlosen begegnet, die Ortschaften namens ?Dazwischen? besucht, der sich mit eigener Kraft von Normalität zur Abweichung und umgekehrt bewegen muss. Berührende Erfahrung.

\section{Fazit}

Inhaltlich genommen eignet sich der vorliegende Band sehr gut als Vertiefungsstudie und zum großen Teil auch als Handbuch in zahlreichen Studienfächern: von Literaturwissenschaften und Kulturwissenschaften über Diskursethik, Wirtschaftsethik bis zur interkulturellen Pädagogik, Gender Studies, philosophischen Anthropologie. Gerade aus der Sicht einer Philosophin scheint die fünffache Botschaft des Bandes besonders aktuell:

1. Die Normen, Regelungen und Normalitäten sind ?Produkte von historischen, sozialen und politischen Prozessen; sie sind 'konstruierte' , historisch bedingte Begriffe, die nie definitiv bestimmt werden können? (S. 11). In jeder Epoche, und ganz besonders in der Epoche von Individualismus, Autonomie und Liberalismus sehnt sich der Mensch nach einem normativen Halt. Doch die Normen und Normalitäten ? als eben ?gesetzt? und ?konstruiert? ? sind in der heutigen dynamischen Epoche mehr denn je verbesserungsbedürftig. Scheitert der ?deliberative Prozess der Entwicklung der Norm? (Schmiedknecht: ?Was bedeutet gesellschaftliche Verantwortung in Zeiten der Globalisierung und des wirtschaftlichen Umbruchs??) oder werden die Individuen von der ?explizit normativen? sozialpolitischen Reformdebatten? (S. Wrobel: ?Es ist notwendig und wir werden es machen. Basta!?) ausgeschlossen, so verlassen sie die Heimat und immigrieren in andere Länder und Kulturen, wo das ?ständige Unterwegs-Sein? (Ch. Baumberger, A.a.O.) doch als besserer Lebensstil erscheint.

2. Der Mensch versteht sich als ein Individuum wie auch als ein Mitmensch. Das Bedürfnis nach der Authentizität, Differenz und Individualisierung ist ebenso primär und stark wie sein Bedürfnis nach Normalität, Identität und Zugehörigkeit. Dies sind zwar unterschiedliche, aber eng miteinander verbundene Aspekte des Lebens. Es wäre völlig falsch zu erwarten (sei es eine persönliche Erwartung, sei es die Erwartung einer gesellschaftlichen Mehrheit oder einer politischen Instanz), dass irgendjemand ein vollkommenes Leben führt, welches nur eines dieser zwei Bedürfnisse erfüllt. Das betrifft alle Ebenen der menschlichen Existenz: von Arbeit und Kreativität (Krämer: ?Abweichung und Norm in der Figur des unternehmerischen Selbst?), über die Sprache (Tinner: ?Normen und Abweichungen in der Zweisprachigkeit?) bis auf die Sexualität (Bettels: ?Heteronormativität in der slowakischen Gesellschaft?).

3. Der Mensch konstruiert gemeinschaftliche (moralische, politische, ökonomische, kulturelle etc.) Normen, Definitionen und Identitäten um einer überschaubaren, sicheren Koexistenz willen. Doch der konstruktivistische Fleiß hat eine paradoxe Nebenfolge: wie der atavistische Naturinstinkt, so grenzt auch das kulturell geprägte ?wir ? ihr? Trennschema die ?Anderen? und ?Fremden? aus. Erst der ?der Umbruch zur Demokratie? (Bettels. A.a.O.) bringt eine Öffnung ?und damit neue Möglichkeiten zur kollektiven Identitätsbildung? (S. 61) ? jedenfalls, wenn der demokratische Umbruch nicht nur die Verfassung, sondern auch die Mentalitäten, Verhaltensweisen und institutionelle Prozeduren erreicht hat.

4. Jedes organisierte, hierarchische (wie flach die Hierarchie auch sein mag) Normensystem kennt die inneren NichtNormalitäten. Diese bezeichnet man üblicherweise als emanzipatorisch, rebellisch oder anarchisch. Demokratie als eine kollektive Selbstbestimmungsform bietet einen Spielraum für progressive Abweichungen, die mit der Zeit zur Normverbesserung beitragen. Dennoch sind die demokratischen Gesellschaften noch nicht für eine uneingeschränkte gastgeberische Rolle vorbereitet: auch hier scheitern nicht selten die Versuche, sich einzuleben (vgl. Baumberger: ?Literaturen der Transmigration: Zsuzsanna Gahse?) und empfangen zu werden. Daraus resultieren immer neue Strategien, Überlappungen und Dialektiken. Sie bilden einen fruchtbaren Grund für Schriftsteller und Künstler, die subtil-perfide Kämpfe zwischen den Grenzwächtern und den Eindringlingen in ihrer eigenen Sprache zum Ausdruck bringen und so die ?Mikrokapilaren des Diskurses? (Foucault) von innen sprengen (vgl. Tinner: ?Normen und Abweichungen in der Zweisprachigkeit ? eine neurolinguistische Analyse?).

5. Die Grenzen, die wir ziehen und passieren, sind durchaus mentaler oder konventioneller Natur. Es ist wichtig, sie nicht stagnieren lassen? denn sie sind nur ?Synthesen des Unmöglichen?, wie Goethe es einst treffend formuliert hatte. 